\title{
Cantilever Beam Vibration Suppression Using Modal Energy Redistribution
}

\author{
Shahin S. Nudehi \\ Mechanical Engineering Department, Valparaiso University, Valparaiso, IN 46383, USA \\ Correspondence should be addressed to Shahin S. Nudehi, shahin.nudehi@valpo.edu \\ Received 30 November 2011; Accepted 17 April 2012 \\ Academic Editor: Luc Gaudiller
}

Copyright () 2012 Shahin S. Nudehi. This is an open access article distributed under the Creative Commons Attribution License, which permits unrestricted use, distribution, and reproduction in any medium, provided the original work is properly cited.

Modal energy redistribution is utilized to suppress vibrations of a cantilever beam. The energy redistribution between the modes of the beam is achieved by switching on/off an end force which causes varying stiffness of the beam. The control methodology to suppress all the modes relies on continuously funneling the energy from the higher to the lower modes with the end force and dissipating the energy associated with the fundamental mode. We present an analytical framework for control design exploiting the modal energy redistribution and verify the results through simulations and experiments.

\section{Introduction}

There have been extensive studies on active vibration of flexible structures in the literatures due to its many applications such as space and terrestrial structures (e.g., [1-3]). In many of these studies, the control scheme for these systems is based on a finite number of modes, and the resulting closed-loop system is prone to instability due to spillover $[4,5]$. There are different ways to avoid spillover in flexile structures. One approach is to have a collocated structure guarantee closed-loop system stability despite model truncation [68]. Another approach to avoid spillover is redistribution of energy in vibrating structures from mode to mode and, in space, from one region of the structure to another $[9,10]$. In the latter approach, stiffness variations of the structure and the difference between modes in two stiffness states are exploited to gain control authority and suppress vibrations. Some examples of stiffness variations in vibration suppression are [11-13] where the stiffness variation and information of the modes are used to reduce energy of the system. This research also uses stiffness variation to redistribute the modal energy and suppress vibration. However, in our study, all the modal states of the structure except the first (fundamental) one are not available or reliable to be used in a feedback closed loop. As a result, displacement and velocity of the structure at different places are used to turn on and off an end force to redistribute the energy of the beam such that higher modes' energy funnel to the lower modes or get suppressed. The energy associated with the first mode is dissipated by employing a feedback closed-loop system. We show that in this scheme that energy of the beam decreases if the switching of the end force (i.e., turning on/off the end force) does not add significant energy to the beam or removes its energy.

This paper is organized as follows. In Section 2, we show a proof of the concept of why modal energy gets redistributed using an end force and how the energy of the beam is reduced. In Section 3, we consider a cantilever beam with an end force and derive its mathematical model. Additionally, we look at the modal energy of the beam and introduce a switching strategy which minimally affects the energy of the beam. Sections 4 and 5 provide simulation and experimental results, respectively. Concluding remarks and future research directions are given in Section 6.

\section{Background and Notation}

2.1. Proof of Concept. A cantilever beam with an end force is shown in Figure 1. The goal is to change the mode shapes of the beam using the end force and exploit these changes in beam vibration suppression. 


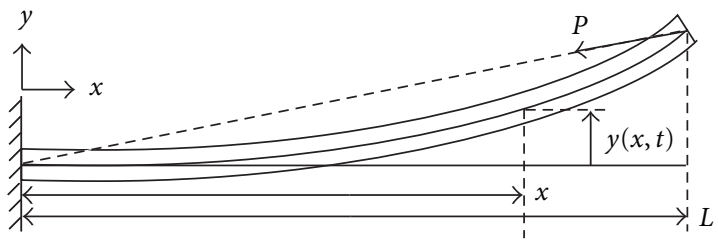

FIgURE 1: A flexible cantilever beam with an end force.

Consider an idealized static problem wherein the end force is instantaneously switched between two values $P_{0}$ and $P_{1}$, and the fundamental modal components (first mode) are repeatedly removed from the system after each switch. We will show that such a strategy removes all the energy from the beam including higher modes. In addition, this strategy only requires that one only be able to suppress the fundamental mode corresponding to $P_{0}$ and $P_{1}$ rather than all the modes of the beam. For the calculations, we denote the attendant mode corresponding to each of mode shapes for $P_{0}$ and $P_{1}$ as $\phi_{j}(x)$ and $\psi_{j}(x)$, respectively. To get an idea how the modes shapes compare with different end forces, in Figure 2 the first four mode shapes of a cantilever beam for $P=0 \mathrm{~N}$ and $P_{1}=40 \mathrm{~N}$ are shown. In this figure, the beam length, width, and thickness are $1 \mathrm{~m}, 0.05 \mathrm{~m}$, and $0.003 \mathrm{~m}$, respectively, with aluminum properties. These dimensions and property are used in the simulation and the experimental section of this paper.

If one starts with a beam deflection $y_{0}(x, t)$ and end-load of $P_{0}$, then we can write

$$
y_{0}(x, t)=\sum_{j=1}^{N} \delta_{j}(t) \phi_{j}(x)
$$

where $N$ denotes the modal truncation level, and $\delta_{j}(t)$ is the $j$ th modal amplitude component. Assuming that one can remove the first mode, the resulting shape is given by

$$
y_{1}(x, t)=y_{0}(x, t)-\delta_{1}(t) \phi_{1}(x)=\sum_{j=2}^{N} \delta_{j}(t) \phi_{j}(x) .
$$

At this point, the end load is switched to $P_{1}$, and the shape is now conveniently expressed as

$$
y_{1}(x, t)=\sum_{j=1}^{N} \beta_{j}(t) \psi_{j}(x)
$$

It is assumed that the first mode is again removed while $P=$ $P_{1}$, resulting in the shape

$$
y_{2}(x, t)=\sum_{j=2}^{N} \beta_{j}(t) \psi_{j}(x)
$$

The end load is then switched back to $P_{0}$ where the shape can then be expressed by

$$
y_{2}(x, t)=\sum_{j=1}^{N} \gamma_{j}(t) \phi_{j}(x) .
$$

This completes one cycle of the process, and one is interested in how the new modal coefficients, the $\gamma_{j}(t)$, are related to the originals, the $\delta_{j}(t)$. This is conveniently described by a linear mapping

$$
\Gamma=M \Delta
$$

where $\Gamma$ and $\Delta$ are the vectors of modal coefficients

$$
\begin{aligned}
& \Gamma=\left(\gamma_{1}, \gamma_{2}, \ldots, \gamma_{n}\right)^{T}, \\
& \Delta=\left(\delta_{1}, \delta_{2}, \ldots, \delta_{n}\right)^{T},
\end{aligned}
$$

and $M$, the mapping matrix, can be developed by a sequence of calculations that use modal projections for each level of the end force as follows:

$$
\begin{aligned}
\gamma_{i}(t)=\left\langle y_{2}(x, t), \phi_{i}(x)\right\rangle & =\left\langle\sum_{j=2}^{N} \beta_{j}(t) \psi_{j}(x), \phi_{i}(x)\right\rangle \\
& =\sum_{j=2}^{N} \beta_{j}(t)\left\langle\psi_{j}(x), \phi_{i}(x)\right\rangle .
\end{aligned}
$$

( $\langle f, g\rangle$ is the inner product of functions $f$ and $g$ denoted by $\left.\int_{0}^{L} f g d x\right)$, and similarly,

$$
\beta_{j}(t)=\sum_{k=2}^{N} \delta_{k}(t)\left\langle\psi_{j}(x), \phi_{k}(x)\right\rangle .
$$

Now, substituting for $\beta_{j}(t)$ from (9) in (8) results in

$$
\begin{array}{r}
\gamma_{i}(t)=\sum_{j=2}^{N} \sum_{k=2}^{N}\left\langle\phi_{i}(x), \psi_{j}(x)\right\rangle\left\langle\psi_{j}(x), \phi_{k}(x)\right\rangle \delta_{k}, \\
i=1, \ldots, N .
\end{array}
$$

Comparing this equation with (6) reveals the structure of the mapping matrix $M$. It should be noticed that the convergence of this process depends on the $N \times N$ linear operator $M$, which can be constructed as follows: the first column contains all zeros since the first modal coefficient was zeroed out (note that this implies that $M$ will always have at least one zero eigenvalue). The remaining columns are filled in by the coefficient $\left\langle\phi_{i}(x), \psi_{j}(x)\right\rangle\left\langle\psi_{j}(x), \phi_{k}(x)\right\rangle, i=1,2, \ldots, N, j, k=$ $2,3, \ldots, N$. If all eigenvalues of $M$ lie inside the unit circle, the process will converge, implying that all modes under consideration die out under repeated cycling and removal of the first relevant mode. In fact, rate of convergence (or divergence) is dictated by these eigenvalues. Figure 3 depicts a schematic of the concept of the modal energy redistribution using the end force.

The above process for the cantilever beam whose mode shapes are shown in Figure 2 is carried out for the first four modes, and the resulting eigenvalues for the mapping matrix $M$ are

$$
\lambda_{i}=\{0,0.51,0.87,0.92\} .
$$

We can see that the rate of convergence for the higher modes is much smaller as compared to the lower modes. In 


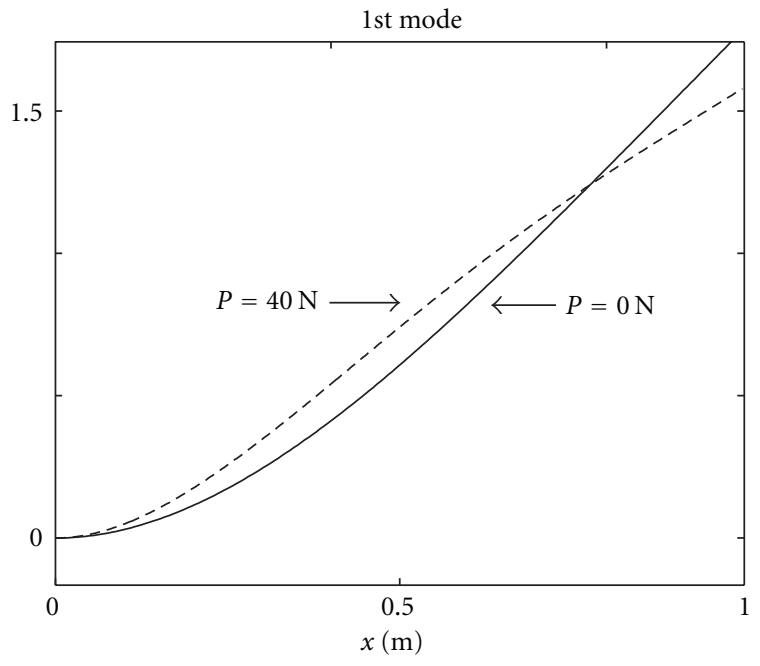

(a)

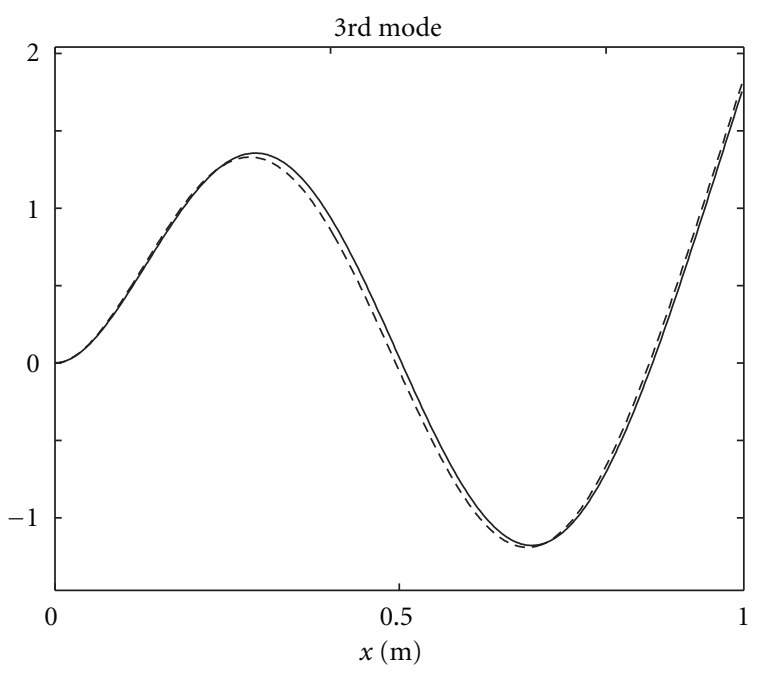

(c)

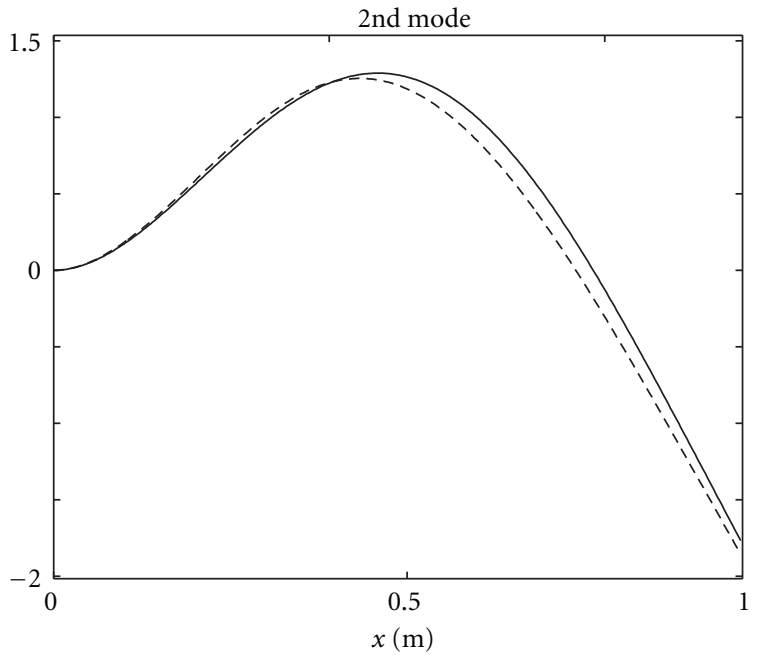

(b)

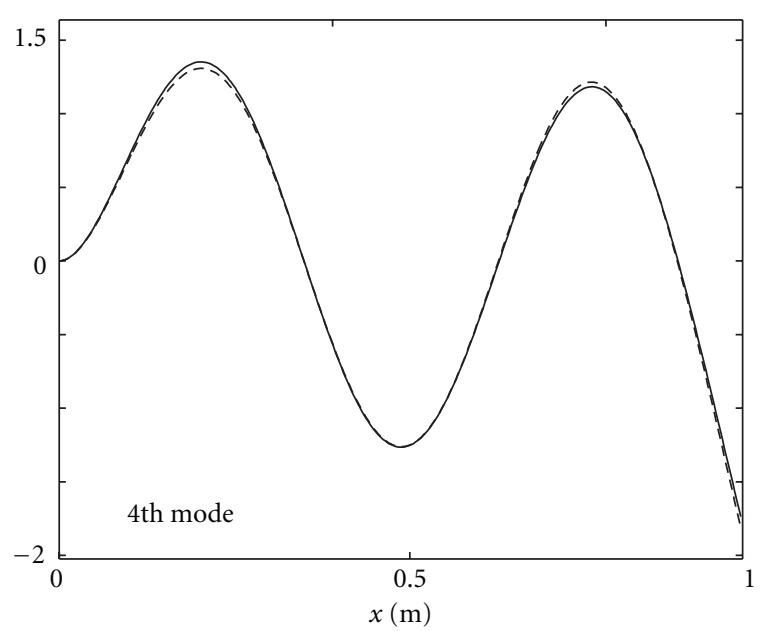

(d)

FIgURE 2: Cantilever beam mode shapes for $P=0 \mathrm{~N}$ and $P=40 \mathrm{~N}$.

other words, the absolute value of the higher modes is close to unity. Note that in this calculation it is assumed that the end force does not add or remove energy to or from the beam in the time interval that is switched on which; this is very unlikely to happen. In the next section, we study a switched system that is comprised of two subsystems (corresponding to the case where the end force is on or off) to model the effect of switching on energy of each modes.

\section{Dynamic Analysis}

3.1. Mathematical Modeling. Consider the cantilever beam of length $L$ and uniform cross-sectional area $A$, shown in Figure 1. Let $P$ be the force acting at the free end of the beam such that the line of action always passes through the fixed end of the beam. Under assumption of Euler-Bernoulli theory and small deflections, the equation of motion of the beam in Figure 1 can be written as follows:

$$
E I y^{\prime \prime \prime \prime}+P y^{\prime \prime}+\rho A \ddot{y}=0,
$$

where $E, I$, and $\rho$ are the Young's modulus of elasticity, area moment of inertia, and density of the beam, respectively, and $y^{\prime}$ and $\dot{y}$ denote the partial derivatives of $y(x, t)$ with respect to $x$ and $t$, respectively. Equation (12) is identical to the equation of a beam with a follower end force [14] and a beam with an axial end force whose line of action remains parallel to the undeformed axis of the beam. However, this configuration is easier and more practical to implement as compared to a follower force. The boundary conditions of the beam in Figure 1 are, however, different from beams with 


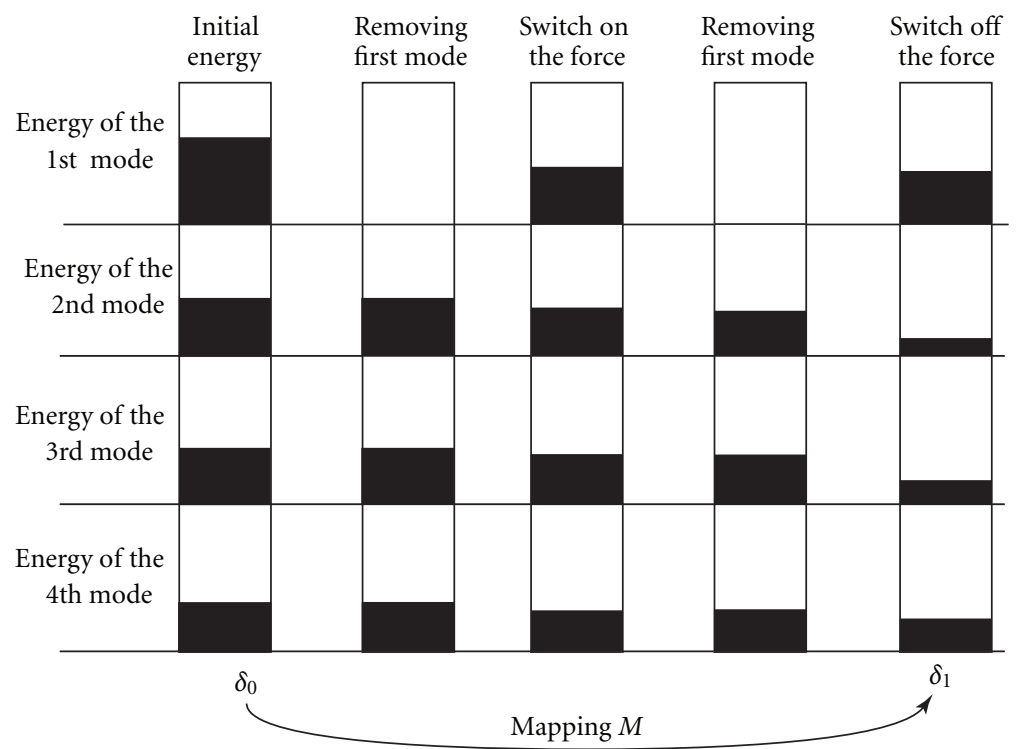

FIGURE 3: Concept of the control strategy to redistribute and remove the beam's energy.

follower and axial end forces. The geometric boundary conditions, which are related to zero deflection and zero slope at the fixed end, are

$$
y(0, t)=0, \quad y^{\prime}(0, t)=0 .
$$

The natural boundary conditions corresponding to zero moment and nonzero shear force at the free end are given by the relations [15]

$$
y^{\prime \prime}(L, t)=0, \quad E I y^{\prime \prime \prime}(L, t)+P\left\{y^{\prime}(L, t)-\frac{1}{L} y(L, t)\right\}=0 .
$$

In order to obtain an approximate solution to (12) subject to the boundary conditions in (13) and (14), we multiply (12) with a weight function $w(x)$ and integrate it over the length of the beam

$$
\begin{gathered}
\int_{0}^{L} E I w(x) y^{\prime \prime \prime \prime}(x, t) d x+\int_{0}^{L} P w(x) y^{\prime \prime}(x, t) d x \\
+\int_{0}^{L} \rho A w(x) \ddot{y}(x, t) d x=0 .
\end{gathered}
$$

We simply assume that $w(x)$ is continuous, twice differentiable and satisfies the two geometric boundary conditions in (13), namely,

$$
w(0)=0, \quad w^{\prime}(0)=0 .
$$

In order to distribute the derivatives equally between $y(x, t)$ and $w(x)$, we integrate the first integral in (15) twice by parts, and the second integral once by parts, to get

$$
\begin{aligned}
& \left.\left(E I w y^{\prime \prime \prime}-E I w^{\prime} y^{\prime \prime}+P w y^{\prime}\right)\right|_{0} ^{L} \\
& \quad+\int_{0}^{L}\left(E I w^{\prime \prime} y^{\prime \prime}-P w^{\prime} y^{\prime}+\rho A w \ddot{y}\right) d x=0 .
\end{aligned}
$$

Equation (17) is called the weak form [16] of (12). Using the natural boundary conditions of $y$ in (14) and the geometric boundary conditions of $w$ in (16), we get

$$
P \frac{w(L) y(L, t)}{L}+\int_{0}^{L}\left(E I w^{\prime \prime} y^{\prime \prime}-P w^{\prime} y^{\prime}+\rho A w \ddot{y}\right) d x=0 .
$$

We now use Rayleigh-Ritz approximation [16] to express $y(x, t)$ as a linear combination of $N$ suitable functions that satisfy the geometric boundary conditions in (13). Specifically, we use the first $N$ normalized mode shapes of the clamped-free cantilever beam, as follows:

$$
y_{N}(x, t)=\sum_{i=1}^{N} \delta_{i}(t) \phi_{i}(x) .
$$

In the above equation, $\phi_{i}(x), i=1,2, \ldots, N$, are the assumed modes, $N$ is the number of desired modes, and $\delta_{i}(t), i=1,2$, $\ldots, N$, are the corresponding modal displacements. Now, by choosing $y(x, t) \approx y_{N}(x, t)$ and $w(x)=\phi_{j}(x), j=1,2, \ldots$, $N$, consistent with (16), we get $N$ differential equations from (18), as follows:

$$
\begin{aligned}
& E I \sum_{i=1}^{N}\left(\int_{0}^{L} \phi_{i}^{\prime \prime} \phi_{j}^{\prime \prime} d x\right) \delta_{i} \\
& \quad-P \sum_{i=1}^{N}\left(\int_{0}^{L} \phi_{i}^{\prime} \phi_{j}^{\prime} d x-\frac{\phi_{i}(L) \phi_{j}(L)}{L}\right) \delta_{i} \\
& \quad+\rho A \sum_{i=1}^{N}\left(\int_{0}^{L} \phi_{i} \phi_{j} d x\right) \ddot{\delta}_{i}=0, \quad j=1,2, \ldots, N .
\end{aligned}
$$

Using the orthogonality property of the assumed mode shapes, the above $N$ equations can be written as follows:

$$
\rho A \ddot{\delta}+(K-P C) \delta=0,
$$


where $\delta=\left(\delta_{1}, \delta_{2}, \ldots, \delta_{N}\right)^{T}, K \in R^{N \times N}$ is a diagonal positive definite matrix with elements $K_{i i}, C \in R^{N \times N}$ is a positive definite symmetric matrix $[17,18]$ with elements $C_{i j}$, and $P$ is assumed to be positive in the direction shown in Figure 1. The elements $K_{i i}$ and $C_{i j}$ have the following expressions:

$$
\begin{gathered}
K_{i i}=E I \int_{0}^{L}\left[\phi_{i}^{\prime \prime}(x)\right]^{2} d x, \\
C_{i j}=\int_{0}^{L} \phi_{i}^{\prime}(x) \phi_{j}^{\prime}(x) d x-\frac{\phi_{i}(L) \phi_{j}(L)}{L} .
\end{gathered}
$$

Equation (21) shows that the end force in Figure 1 is conservative in nature. This can now be claimed [19] from the symmetric nature of the matrix $(K-P C)$. Additionally, when $P=0$, the eigenvalues of $(K-P C)$ are the same as the eigenvalues of $K$, which are all positive. As $P$ is increased, all the eigenvalues start moving towards the origin before crossing the imaginary axis and becoming negative. This can be claimed from the physics of the buckling problem and has also been verified using numerical simulations. In the derivation of (21), structural damping was assumed to be absent. If structural damping is present, we can use

$$
\rho A \ddot{\delta}+D \dot{\delta}+(K-P C) \delta=0,
$$

where $D \in R^{N \times N}$ is a diagonal positive definite matrix of modal damping.

3.2. Switching Strategy and Stability Proof. The goal of this section is to introduce a switching strategy for the end force $P$ to funnel energy from the higher modes of vibrations to the first mode with minimal contribution to the total energy of the system. The following shows (23) modified for a beam subjected to the end load $P$ with a control law to remove the first mode of vibration:

$$
\rho A \ddot{\delta}+D \dot{\delta}+(K-P(t) C) \delta+H_{1}\left(\delta_{1}, \dot{\delta}_{1}\right)=0 .
$$

Here, $P(t)$ is zero $\left(P_{0}=0\right)$ when the end force is switched off and is equal to $\left(P_{1}<P_{\text {buckling }}\right)$ when the force is switched on, and $H_{1}$ is the diagonal control law to remove the oscillations of the fundamental frequency. By expanding this equation and multiplying the first row by $\dot{\delta}_{1}$, the second row by $\dot{\delta}_{2}$, and so on, we have

$$
\begin{aligned}
& \frac{d E_{1}}{d t}=-\dot{\delta}_{1} D_{11} \dot{\delta}_{1}-\dot{\delta}_{1} h_{1}\left(\dot{\delta}_{1}, \delta_{1}\right)+P \dot{w}_{1}\left(\dot{\delta}_{1}, \delta\right) \\
& \frac{d E_{2}}{d t}=-\dot{\delta}_{2} D_{22} \dot{\delta}_{2}+P \dot{w}_{2}\left(\dot{\delta}_{2}, \delta\right) \\
& \vdots \\
& \frac{d E_{n}}{d t}=-\dot{\delta}_{n} D_{n n} \dot{\delta}_{n}+P \dot{w}_{n}\left(\dot{\delta}_{n}, \delta\right)
\end{aligned}
$$

where $E_{i}=(1 / 2)\left(\rho A \dot{\delta}_{i}^{2}+K_{i i} \delta_{i}^{2}\right)$ is the modal energy corresponds to the $i$ th mode, $\dot{w}_{i}\left(\dot{\delta}_{i}, \delta\right)=\dot{\delta}_{i}\left[C_{i}\right] \delta, D_{i i}$ is the $i$ th element of the $[D]$ matrix, and $\left[C_{i}\right]$ refers to the $i$ th row of the $[C]$ matrix.
Summing up all the rows of (25) results in

$$
\begin{aligned}
& \frac{d}{d t}\left(E_{1}+E_{2}+\cdots+E_{n}\right) \\
& =-\sum_{i=1}^{n} \dot{\delta}_{i} D_{i i} \dot{\delta}_{i}+P \sum_{i=1}^{n} \dot{w}_{i}\left(\dot{\delta}_{i}, \delta\right)-\dot{\delta}_{1} h_{1}\left(\dot{\delta}_{1}, \delta_{1}\right) .
\end{aligned}
$$

In (26), the term $-\sum_{i=1}^{n} \dot{\delta}_{i} D_{i i} \dot{\delta}_{i}$ is negative. In addition, the control law for the fundamental mode is assumed to be designed properly and also removes energy of the first mode, that is, $-\dot{\delta}_{1} h_{1}\left(\dot{\delta}_{1}, \delta_{1}\right)<0$. As a result, as long as the end force is turned on, whenever the value of $\dot{W}_{P}=\sum_{i=1}^{n} \dot{w}_{i}\left(\dot{\delta}_{i}, \delta\right)=0$, the total energy of the system will be reducing, and the endload does not affect the total energy of the beam. In fact, one could also reduce the energy of the beam further by turning the end force on when it does negative mechanical work [17], that is, $\dot{W}_{P}<0$. The work of the end force (per unit time), $\dot{W}_{p}$, consists of two terms [20]:

$$
\dot{W}_{P}=P \int_{0}^{L} \frac{\partial \dot{y}(x, t)}{\partial x} \frac{\partial y(x, t)}{\partial x} d x-\frac{P \dot{y}(L, t) y(L, t)}{L} .
$$

The first integral term represents the work done (per unit time) by the axial component of the end force $P$ against the change in the length of the beam due to elastic deformation [20], and the second component is the work done by the lateral component of the force $P$ at the tip of the beam due to lateral motion of the beam. It should be noted that due to small deformation of the beam, the work done by the lateral component of the force $P$ is a magnitude of order smaller than the axial component. As a result,

$$
\dot{W}_{P} \cong P \int_{0}^{L} \frac{\partial \dot{y}(x, t)}{\partial x} \frac{\partial y(x, t)}{\partial x} d x
$$

From (28), one can calculate the work done by the end force $P$ at every instant using information of the displacement and velocity of every location along the beam. In practice, (28) can be discretized to consider a finite number of location displacements and velocities to calculate the work done by $P$ as seen in the following:

$$
\dot{W}_{\mathrm{P}} \approx P \sum_{i=0}^{i=r-1} \frac{\left[\dot{y}\left(x_{i+1}, t\right)-\dot{y}\left(x_{i}, t\right)\right]\left[y\left(x_{i+1}, t\right)-y\left(x_{i}, t\right)\right]}{x_{i+1}-x_{i}} .
$$

Here, $r$ is the number of locations used to calculate the integral, $x_{i}$ is the position at location $i=0 \cdots r-1$, and $y\left(x_{i}, t\right)$ and $\dot{y}\left(x_{i}, t\right)$ are the displacement and velocity of the beam at location $x_{i}$, respectively. Equation (29) shows that the displacement and velocity information of the beam at different locations can be used to estimate the work done by the end force $P$ without information of the modal state estimations. In other words, one can use the end force to redistribute or remove the modal energy without knowledge of the modes or number of modes involved in the beam's oscillations. 
TABLE 1

\begin{tabular}{ll}
\hline Material & Aluminum \\
Young's modulus & $70 \mathrm{GPa}$ \\
Mass density & $2730 \mathrm{~kg} / \mathrm{m}^{3}$ \\
Dimensions & $1.00 \times 0.05 \times 0.003 \mathrm{~m}$ \\
\hline
\end{tabular}

\section{Simulation Results}

In this section, the efficacy of the idea of redistributing the modal energy with an end force is investigated using simulation results. We assumed the material and geometric properties of the beam to be as shown in Table 1 .

For a four-mode approximation of beam dynamics, the $K$ and $C$ matrices of our mathematical model in (22) were computed as

$$
\begin{aligned}
K & =\left(\begin{array}{cccc}
97.3 & 0 & 0 & 0 \\
0 & 3824.4 & 0 & 0 \\
0 & 0 & 29976.5 & 0 \\
0 & 0 & 0 & 115111.1
\end{array}\right), \\
C & =\left(\begin{array}{cccc}
0.65 & -3.38 & -0.06 & -2.59 \\
-3.38 & 28.42 & -18.35 & 9.58 \\
-0.06 & -18.35 & 73.30 & -31.65 \\
-2.59 & 9.58 & -31.65 & 138.90
\end{array}\right) .
\end{aligned}
$$

The square roots of the diagonal entries of the $K / \rho A$ matrix are the natural frequencies of the beam and are equal to $15.42,96.63,270.6$, and $530.2 \mathrm{rad} / \mathrm{s}$, respectively.

In the first simulation, to better illustrate energy redistribution between the modal states with an end force, we assume the modal damping to be absent, that is, the diagonal entries of the $D$ matrix in (23) are zero. Additionally, the control law to remove the first mode oscillations is also considered to be $h_{1}\left(\dot{\delta}_{1}, \delta_{1}\right)=0$. We used the exact expression for the $\dot{W}_{P}$ to turn on/off the end force to avoid changing the total energy of the beam (i.e., $\dot{W}_{P}=0$ ) after the switchings. We also assume that $P_{1}$ in (24) is selected to be $40 \mathrm{~N}$ which is less than the critical buckling load $(77 \mathrm{~N})$ of the beam. The simulation results are shown in Figure 4 for the following initial conditions in metric units:

$$
\begin{aligned}
& \delta(0)=[0,-0.5,0,0.1]^{T}, \\
& \dot{\delta}(0)=[0,-1.2,0,3.5]^{T} .
\end{aligned}
$$

This simulation shows clearly that the end force causes modal energy redistribution. The oscillations of the beam consist of the first and third modes, although the initial conditions for these two modes are chosen as zero. Clearly, the end force causes energy associated with the second and fourth modes to be redistributed among all the modes of the beam with nonzero end force. It should be noted that the energy transferred by the fourth mode to the other modes is considerably smaller compared to the second mode. This was expected from the values of the mapping matrix from (11).

In the second simulation, the end force $P$ is used for two purposes: to redistribute the modal energy between the modes and to remove the modal energy by doing negative

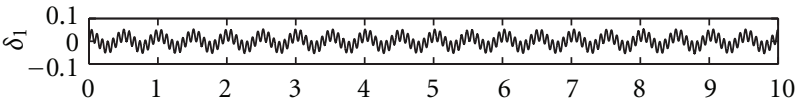

(a)

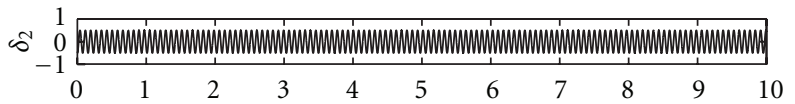

(b)

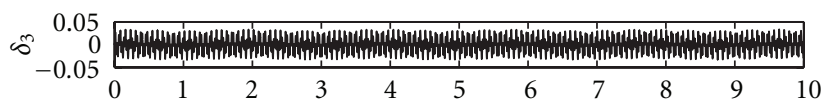

(c)

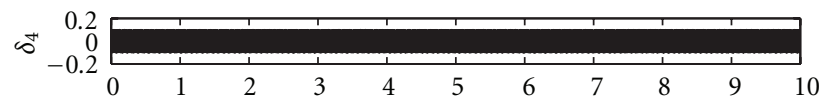

(d)

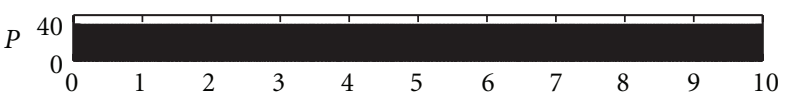

(e)

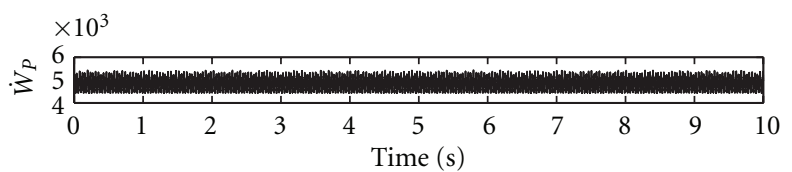

(f)

Figure 4: Modal redistribution of the beam with the switching end force.

work, that is, $\dot{W}_{P} \leq 0$. This is to address the issue of a small percentage of the higher modes energy that gets funneled to the lower modes. We assume that modal states of the beam are not observable except for the first mode and information of the displacement and velocity is used to determine the work of the end force using (29). The control law to remove the first mode oscillation is chosen as $h_{1}\left(\dot{\delta}_{1}, \delta_{1}\right)=2 \dot{\delta}_{1}$, and the modal damping is assumed to be absent as well. To calculate $\dot{W}_{P}$ from (29), velocities and displacement at the base, the midpoint, and the end point locations of the beam are used

$$
\begin{aligned}
\dot{W}_{P} \approx P( & \frac{[\dot{y}(L, t)-\dot{y}(L / 2, t)][y(L, t)-y(L / 2, t)]}{L / 2} \\
+ & \left.\frac{[\dot{y}(L / 2, t)-\dot{y}(0, t)][y(L / 2, t)-y(0, t)]}{L / 2}\right),
\end{aligned}
$$

where $\dot{y}(0, t)=y(0, t)=0$. In Figure 5, the simulation results are plotted for arbitrary initial conditions in SI units

$$
\begin{aligned}
& \delta(0)=[0.3,-0.5,-0.1,0.1]^{T}, \\
& \dot{\delta}(0)=[-0.3,-1.2,0.2,3.5]^{T} .
\end{aligned}
$$

This figure shows the modal amplitudes and energy of the beam rapidly decaying to zero, as expected, but the control 


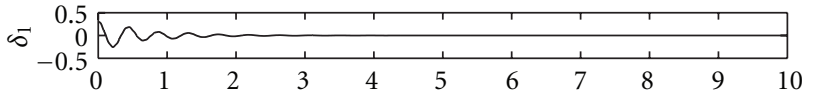

(a)

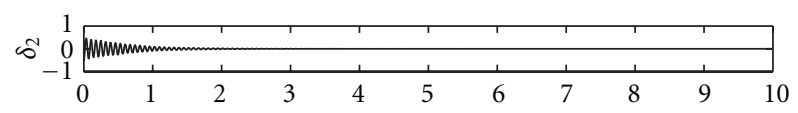

(b)

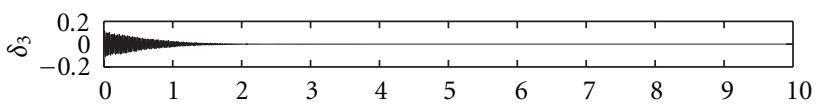

(c)

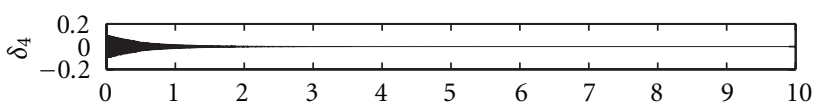

(d)

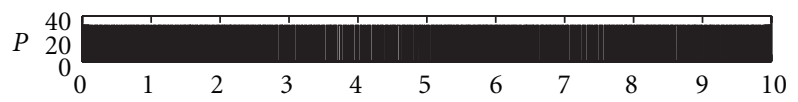

(e)

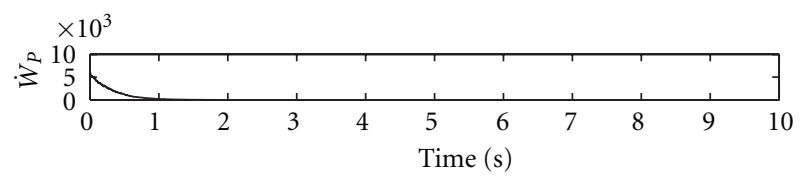

(f)

FIgure 5: Modal redistribution and vibration suppression of the beam with the switching end force and the control law.

input continues switching. This can be attributed to small numerical errors causing frequent change in sign of $\dot{W}_{P}$. This problem can be easily rectified during implementation. It should be noted that the approximation of $\dot{W}_{P}$ in (33) causes at some tiny time intervals that the end force adds energy to the beam instead of redistributing or removing it. This can be resolved by adding more terms (i.e., displacement and velocities measurements) to approximate $\dot{W}_{P}$ in (29). In this result, the beam's energy is dissipated by the control law $h_{1}\left(\dot{\delta}_{1}, \delta_{1}\right)=2 \dot{\delta}_{1}$ and the negative work of the end force $P$.

To see better the relative influence of the end force negative work and the control law in the modal attenuation, another simulation is performed in which the control law is set to zero, $h_{1}\left(\dot{\delta}_{1}, \delta_{1}\right)=0$, and the end force $P$ is used for both modal distributions and modal attenuation. The simulation results are shown in Figure 6 for the same initial conditions used in (33).

These results show that the end force $P$ is capable of removing all the modal energy since $\dot{W}_{P} \leq 0$ at the times the end force is on. In this specific problem, the switching end force can be used solely to remove all the modes without needing the control law $h_{1}\left(\dot{\delta}_{1}, \delta_{1}\right)$. However, in practice, adding the control law makes the attenuation faster as seen in Figures 5 and 6 and (26). More importantly, it could remove any addition in the modal energy due to end force switching time not being exact, which may happen due to the approximation in (32) or a slow response time of the actuator used

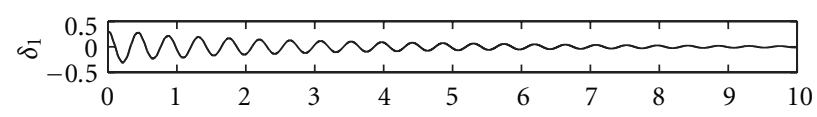

(a)

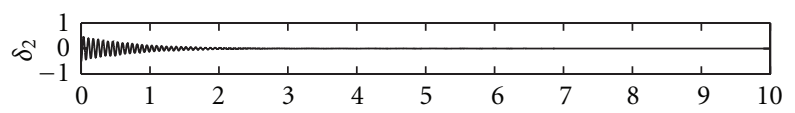

(b)

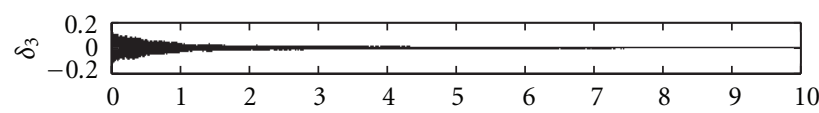

(c)

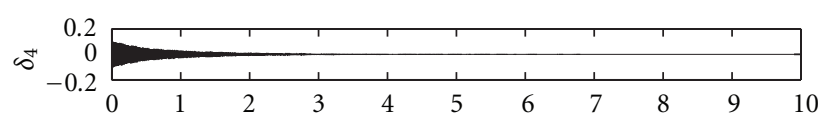

(d)

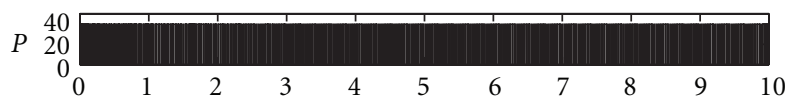

(e)

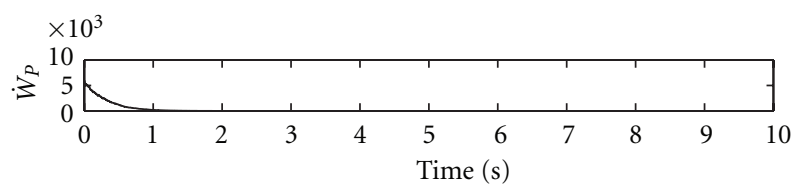

(f)

FIGURE 6: Modal redistribution and vibration suppression of the beam with just the switching end force.

for turning on/off the end force. It should be noted that the bandwidth of the actuator used to switch the end force should be larger than the highest frequency in which $\dot{W}_{P}$ sign changes. If the actuator does not have the requisite bandwidth, there is no guarantee that the end force removes energy of the beam.

\section{Experimental Verification}

We conducted an experiment with a cantilever beam to prove the principal of redistributing and removing modal energies with an end force. In our experimental setup, the end force is applied with a Kevlar cable, wrapped around a pulley at the front face of the beam and then tied to a high-bandwidth servo DC motor [21] shaft as shown in Figure 7.

The motor was driven by a National Instruments NI 9505, brushed DC drive module [22] to pull or release the cable which causes the end force to turn on/off. Two miniature single-axis accelerometers from PCB Electronics [23] are mounted on the midpoint and end point of the beam to derive the displacement and velocity of the beam at these two locations. In the experiment, we did not implement the control law (i.e., $h_{1}\left(\dot{\delta}_{1}, \delta_{1}\right)=0$ ) and only used the end force to redistribute/attenuate the modal energy. (One can easily implement the control's law using a piezoelectric sensor and actuator attached to the beam; see, for instance, [24]). 


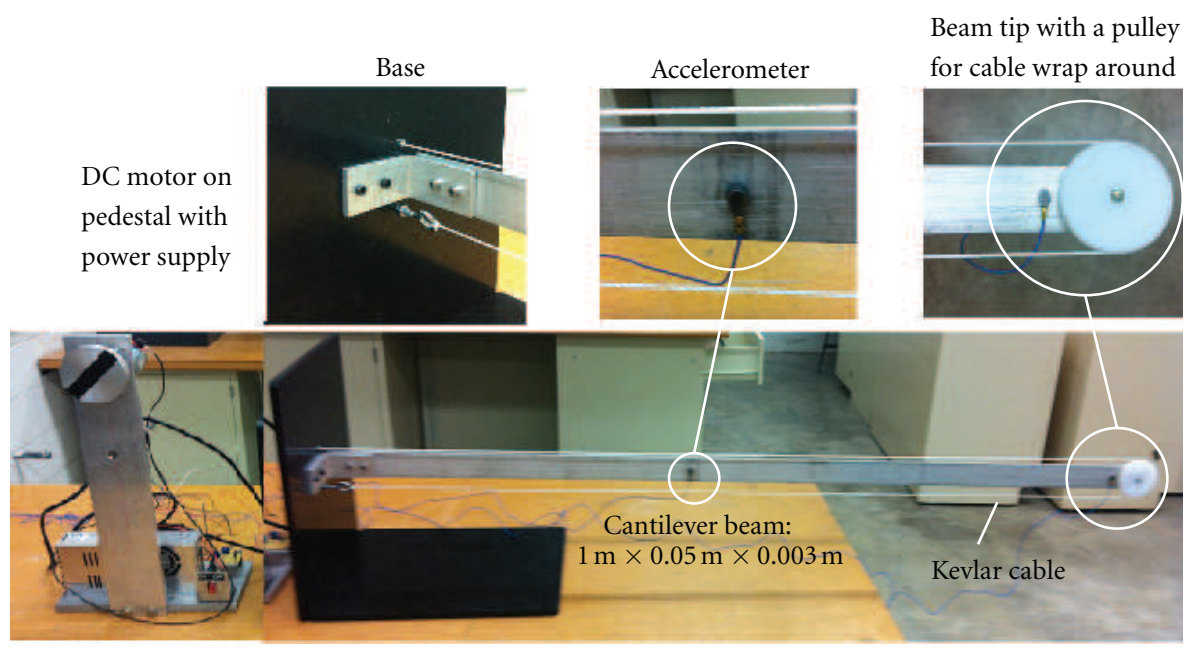

FIGURE 7: Experimental setup.

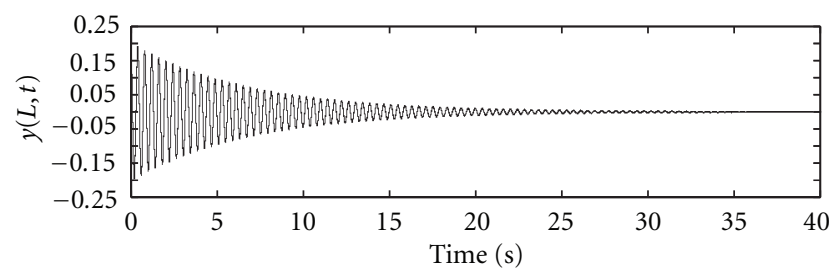

FIGURE 8: Experimental results: free vibration.

We programmed the switching law and the integration processers to achieve displacements and velocities in the LabVIEW FPGA [22] environment and downloaded it to our CompactRIO 9022 processing unit (not shown). The flexible beam and material used in the experiment are the same as the ones used in the simulations presented in Section 4.

The first experiment pertains to free vibration of the beam in the first mode. The time history for the displacement of the beam's tip is shown in Figure 8 in metric units. In the absence of the switching end force, the beam vibration is attenuated slowly by the structural damping. The time required for the amplitude of vibration to decay by a factor of 40 approximately is 25 seconds.

The second experiment pertains to vibration suppression of the beam with the switching end force. The time history for the displacement and velocity of the beam's tip and the end force in the experiment are shown in Figure 9 in metric units. In these results, the initial condition is achieved by deflecting the beam arbitrarily. These results indicate that the control strategy attenuates all the modes of vibration which corroborates our earlier simulation results. It should be noted that the structural damping also contributes to the removal of energy from the beam especially at higher frequencies. In fact, the modal damping of the structure increases whenever the end force is switched on due to an increase in axial stress of the beam [15] which further helps vibration suppression of the beam.

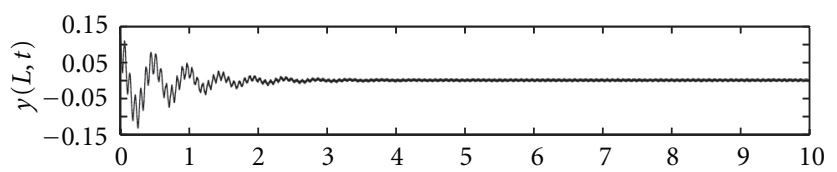

(a)

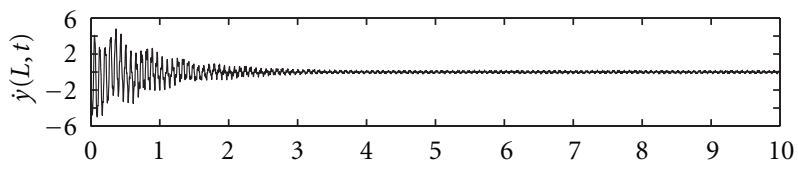

(b)

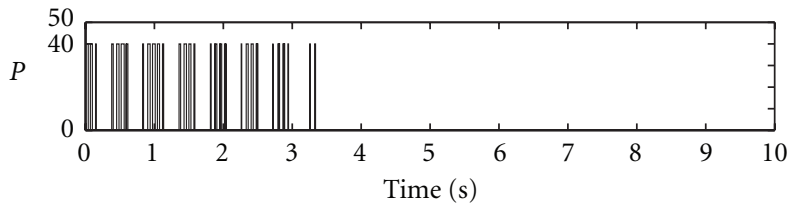

(c)

FIGURE 9: Experimental results: vibration suppression using the end force.

\section{Conclusions}

Modal redistribution and vibration suppression of a cantilever beam with an end force is mathematically studied and experimentally verified. We showed that it is possible to redistribute modal energy of the beam by changing its stiffness using an end force. In addition, the end force was used to suppress the vibrations of the beam since mathematical and simulation results showed that the rate of energy distribution from higher modes to the lower modes of the beam is small. As a result, the end force was also used to remove the energy of the beam. The end force was turned on whenever it either removed or did not change the modal energy of the beam; otherwise, it was turned off. For this purpose, we used lateral displacements and velocities of the beam at different locations to construct and estimate the rate 
of the mechanical work done by the end force without information of the modal amplitudes. Our goal for future work is to employ modal energy redistribution for two- and threedimensional flexible structures in which the stiffness changes with cables at multiple locations along the structure.

\section{Acknowledgments}

The author would like to thank Professor Ranjan Mukherjee of Michigan State University for his useful comments and suggestions during the course of this research. In addition, the author gratefully acknowledges the support provided by Valparaiso University's Creative Work and Research Committee that made this research possible.

\section{References}

[1] D. C. Hyland, J. L. Junkins, and R. W. Longman, "Active control technology for large space structures," Journal of Guidance, Control, and Dynamics, vol. 16, no. 5, pp. 801-821, 1993.

[2] G. S. Nurre, R. S. Ryan, H. N. Scofield, and J. L. Sims, "Dynamics and control of large space structures," Journal of Guidance, Control, and Dynamics, vol. 7, no. 5, pp. 514-526, 1984.

[3] M. J. Balas, "Trends in large space structure control theory: fondest hopes, wildest dreams," IEEE Transactions on Automatic Control, vol. 27, no. 3, pp. 522-535, 1982.

[4] M. J. Balas, "Active control of flexible systems," Journal of Optimization Theory and Applications, vol. 25, no. 3, pp. 415436, 1978.

[5] M. J. Balas, "Feedback control of flexible systems," IEEE Transactions on Automatic Control, vol. 23, no. 4, pp. 673-679, 1978.

[6] J. J. Dosch, D. J. Inman, and E. Garcia, "Self-sensing piezoelectric actuator for collocated control," Journal of Intelligent Material Systems and Structures, vol. 3, no. 1, pp. 166-185, 1992.

[7] D. Halim and S. O. R. Moheimani, "Spatial resonant control of flexible structures-application to a piezoelectric laminate beam," IEEE Transactions on Control Systems Technology, vol. 9, no. 1, pp. 37-53, 2001.

[8] E. H. Anderson, N. W. Hagood, and J. M. Goodliffe, "Modelling of piezoelectric actuator dynamics for active structural control," in in Proceeding of the AIAA/ASME/ASCE/AHS Structures, Structural Dynamics, Materials Conference, pp. 2241-2255, Dallas, Tex, USA, April 1992.

[9] A. Diaz and R. Mukherjee, "Modal disparity enhancement through optimal insertion of nonstructural masses," Structural and Multidisciplinary Optimization, vol. 31, no. 1, pp. 1-7, 2006.

[10] A. R. Diaz and R. Mukherjee, "A topology optimization problem in control of structures using modal disparity," Journal of Mechanical Design, vol. 128, no. 3, pp. 536-541, 2006.

[11] J. Issa, R. Mukherjee, A. R. Diaz, and S. W. Shaw, "Modal disparity and its experimental verification," Journal of Sound and Vibration, vol. 311, no. 3-5, pp. 1465-1475, 2008.

[12] A. Kurdila, W. Clark, W. Wang, and D. McDaniel, "Stability of a class of piezoelectric state-switching methods," in Adaptive Structures and Material Systems-2000, pp. 509-517, American Society of Mechanical Engineers, New York, NY, USA, 2000.
[13] A. Ramaratnam and N. Jalili, "A switched stiffness approach for structural vibration control: theory and real-time implementation," Journal of Sound and Vibration, vol. 291, no. 1-2, pp. 258-274, 2006.

[14] V. Bolotin and G. Herrmann, Nonconservative Problems of the Theory of Elastic Stability, vol. 1991, Pergamon Press, London, UK, 1963.

[15] S. R. M. Nudehi, Active Vibration Suppression of Flexible Structures Using Switching Control [Ph.D. thesis], Michigan State University, East Lansing, Mich, USA, 2005.

[16] J. Reddy, An Introduction to the Finite Element Method, vol. 2, McGraw-Hill, New York, NY, USA, 1993.

[17] S. Nudehi, R. Mukherjee, and S. W. Shaw, "Active vibration control of a flexible beam using a buckling-type end force," Journal of Dynamic Systems, Measurement and Control, vol. 128, no. 2, pp. 278-286, 2006.

[18] H. P. Lee, "Divergence and flutter of a cantilever rod with an intermediate spring support," International Journal of Solids and Structures, vol. 32, no. 10, pp. 1371-1382, 1995.

[19] M. A. Langthjem and Y. Sugiyama, "Optimum design of cantilevered columns under the combined action of conservative and nonconservative loads. Part I: the undamped case," Computers and Structures, vol. 74, no. 4, pp. 385-398, 2000.

[20] S. Rao, Vibration of Continuous Systems, Wiley, New York, NY, USA, 2007.

[21] http://faulhaber group.com/.

[22] http://www.ni.com/.

[23] http://www.pcb.com/.

[24] S. S. Nudehi and R. Mukherjee, "Enhancing controllability and observability in underactuated and undersensed systems through switching: application to vibration control," Journal of Dynamic Systems, Measurement, and Control, vol. 126, no. 4, pp. 790-799, 2004. 

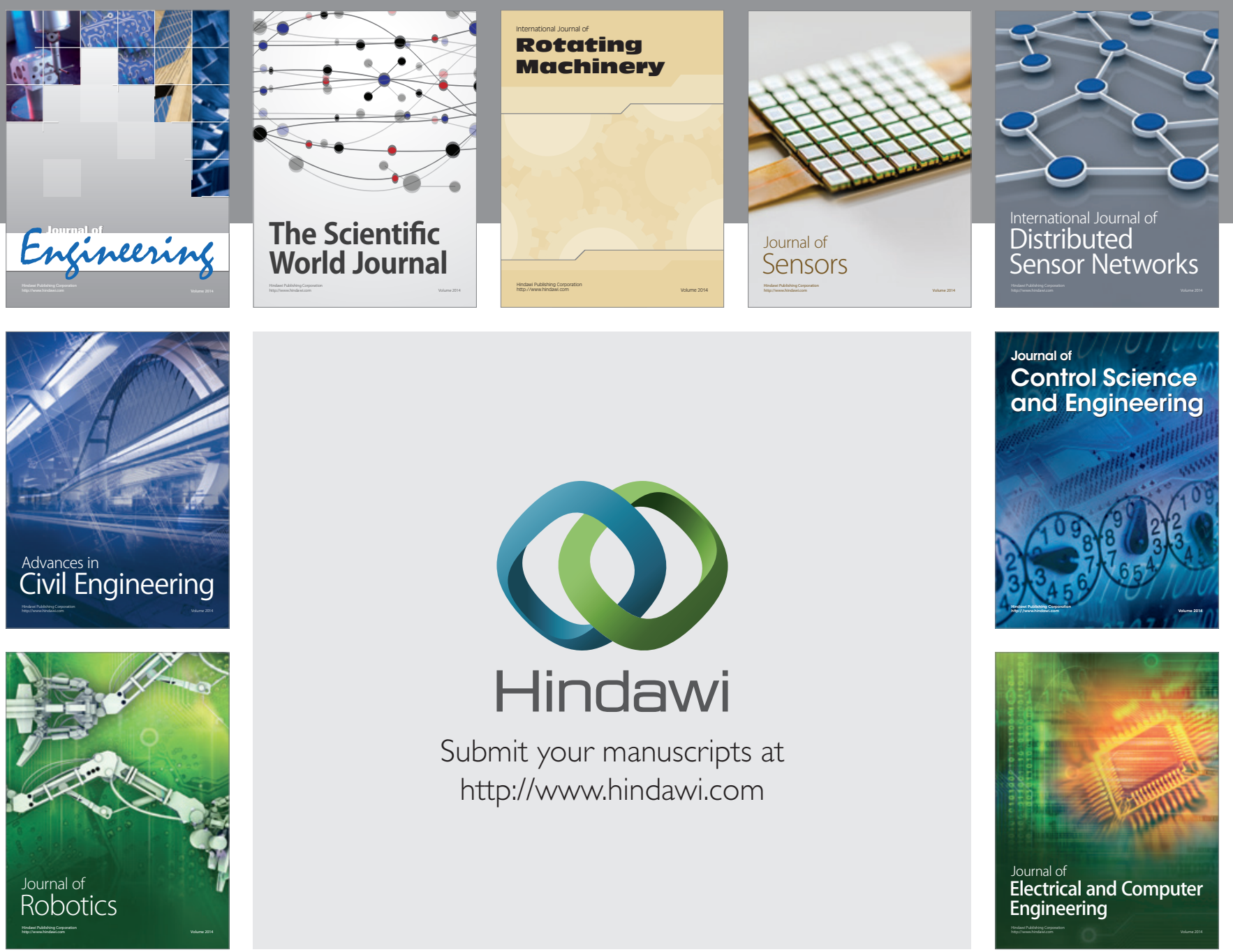

Submit your manuscripts at

http://www.hindawi.com
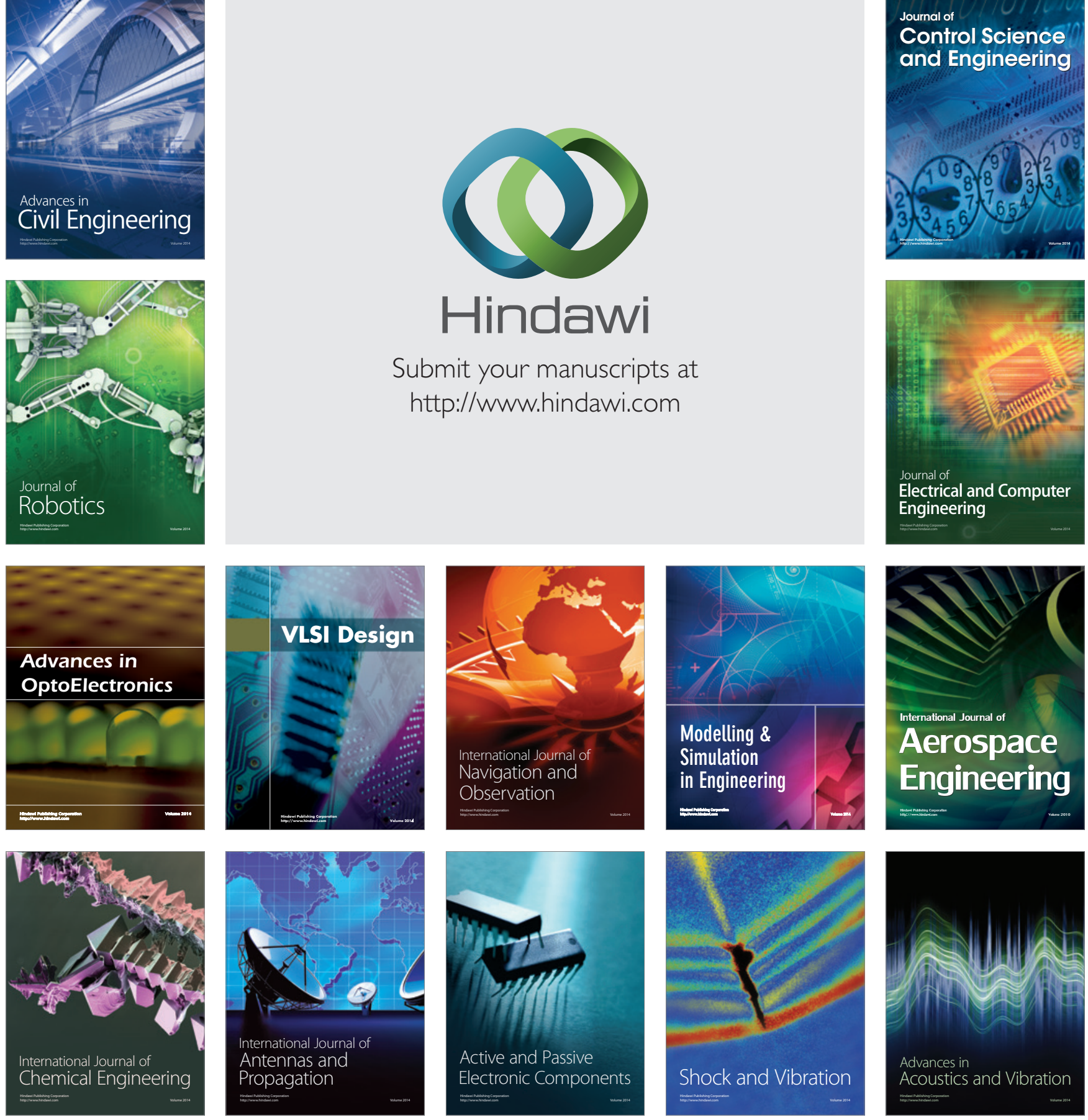